\title{
Dissipation in computation
}

SIR-All proposals for 'reversible' or dissipation-free computation' seem to me to have a common flaw: the discussion concentrates on what happens between the input and output of a gate (or a computer), but neglects what occurs at input and output.

Taking the billiard-ball model as an example, to initiate its operation requires the simultaneous start at $t_{0}$ of several balls from their respective input channels (which determine the shooting direction). To this end the balls have to be inserted some time before $t_{0}$ and held in place against thermal motion. This can be achieved by static friction between balls and tubes or by some kind of bolts placed in front of the balls and held in place by static friction so that they cannot give way prematurely by their own thermal motion. Equally, the driving springs have to be loaded before $t_{0}$ and secured behind the balls by appropriate bolts. Even neglecting all the energy dissipated during these preparations, to initiate our computation at $t_{0}$, we have to withdraw all the bolts against static friction. This has to be done rapidly, or else $t_{0}$ would be indeterminate, and so unavoidably we have to dissipate at least several $k T$ of energy per input ball.

Equal amounts of energy have of necessity to be dissipated at every output. Any gadget that does not yield a recognizable output signal cannot be considered to be a valid computer model; a billiard ball that is elastically reflected at the 'output' without any loss of energy cannot yield any kind of output. Whether the balls, running against reflecting springs, are trapped at the reversal point by appropriate bolts - so that they can serve for readout before they are let loose on their return trip - or whether they shift small signal-bolts at the output (which can be identical to the input-bolts of a subsequent gate), one cannot avoid the dissipation of several $k T$ of energy per bit.

As for those models in which it is claimed that frictional losses could be reduced at will by simply reducing the speed of the balls correspondingly, when the forward-directed motion becomes slow compared with the average thermal motion, the passage of the balls from input to output becomes completely unpredictable; such a device is surely not a valid model for a computer.

Landauer' describes a Fredkin gate in which a ball pushes the two halves of a split control pipe apart. This ball must exert pressure on these control plates, and so will unavoidably experience static friction proportional to this pressure and independent of its velocity during its passage through the control pipe. The ideal viscous fluid', in which the whole affair is assumed to be immersed, will have no effect; it will simply be driven away from between ball and plates. How can such a device be claimed to function without minimum energy dissipation?

Finally, I do not believe that 'unwinding the program' can reduce the energy dissipation that occurs when resetting the output register. No ball can arrive at the output that was not started somewhere as an input ball; therefore, the numbers of input and output balls being equal, it can make no difference whether we discard these balls from the output register or, after unwinding the program, from the input register. Landauer's proposal to avoid resetting losses altogether by (hopefully) dissipation-free copying and uncopying of the input is nothing but the simple transmission from the register into a store of (necessarily) infinite capacity. Now consider the energy required to produce all this hardware.

\section{Taunusstrasse 67}

E. BiedermanN

\section{Böblingen, FRG}

LANDAuer REPLies-Reversible computation tends to be counterintuitive at first exposure. Since its original description ${ }^{2}$, the concept has been elaborated by many with differing viewpoints, including R. P. Feynman ${ }^{3}$. Despite this widespread acceptance, criticism still appears in print, typically without citation of earlier similar debates. My rebuttal ${ }^{4}$ of such a critique cites some of these previous debates. There are various possible realizations of reversible computations described in the literature: Biedermann focuses exclusively on the billiard-ball and Fredkin-gate versions. It is conceivable that one or other of the existing embodiments is flawed: demolishing one version is not an adequate rebuttal to reversible computation itself.

Biedermann focuses on the input and output operations in the billiard-ball model. As I have stressed in ref. 4, information transfer at input and output need not differ from that occurring within the computer. On the other hand, bit transfer at either end can easily be made more dissipative than that within the computer. Biedermann discusses such alternatives but does not tell us why he considers them to be optimal processes.

Most, but not all, reversible computers move back and forth in a diffusive fashion if watched over a short period, but with a predictable velocity over a long period, analogous to that of a brownian particle moving in a force field. Biedermann claims that "such a device is surely not a valid model for a computer". In my opinion, a system that carries out a computation is a computer. If the computer is going to dissipate much less energy than in current technology, it is likely to differ substantially from conventional systems.

Biedermann's charges that I ignore static friction can be answered on four levels. First, we are following the timehonoured example in the discussion of thermodynamic cycles. Second, not all reversible computers depend on mechanical devices; Bennett ${ }^{5}$ has proposed one based on genetic-code machinery, and Likharev's ${ }^{6.7}$ version uses Josephson junctions. Third, in these discussions, we inquire about the limits imposed by physics, and not about what can be practically realized. We are not far from the capability to build devices with atomic precision and with perfectly periodic surfaces, preserved by operation at low temperatures. There will then be no static friction. Last, even in the absence of a lubricating film, and with irregular surfaces, I believe that there is a wide range of conditions that eliminate static friction, as long as the velocity is controlled (rather than the force) and is kept small relative to thermal velocities. For ordinary macroscopic devices such a velocity is uselessly small.

Biedermann points out that the number of information-bearing degrees of freedom is conserved during reversible computations. Both the number of 'balls' and the information in the output reflects that in the input. But in the input most of the information is in the form of a supply of balls in standardized states; the variable information is confined to the program. After a long computation the final state of a reversible computer has many more balls whose state depends on the computation that has occurred.

ROLF LANDAUER IBM Thomas J. Watson Research Center, PO Box 218, Yorktown Heights,

New York 10598, USA

\footnotetext{
1. Landauer, R. Nature 335, 779-784 (1988)

Bennett, C. H. IBM J. Res. Dev. 17, 525-532 (1973)

Feynman. R. P. Found. Phys. 16, 507-531 (1986)

Landaver. R. Found. Phys. 19. $729-732$ (1989).

Bennett, C. H. Int. J. theor. Phys. 21. $905-940$ (1982).

Likharev, K. K. Int. J. theor. Phys. 21, 311-326 (1982).

Likharev, K. K., Rylov, S. V. \& Semenov, V. K. IEEE Trans. Magn. 21, $947-950(1985)$
}

\section{Space sickness on Earth}

SIR-We report here the surprising aftereffects of prolonged centrifuge runs in which we, the three scientist-astronauts on board the D-1 Spacelab mission (US Space Shuttle Challenger flight, 30 October-6 November 1985), have participated. We think we can simulate the space adaptation syndrome, better known as space sickness, on Earth. Such a method has, to our knowledge, not previously been reported, nor has a correlation been found between individual susceptibility to space sickness and Earth-bound motion sickness. For these reasons, it has been difficult to study the cause of space sickness or to develop preventive measures 